Int. J. Electrochem. Sci., 12 (2017) $6092-6107$

\title{
Mercury(II) Trace Detection Using a Glassy Carbon Electrode Functionalized by Chemically Prepared Gold Nanoparticles. Influence of Coating Process on Surface Reactivity and Analytical Performances
}

\author{
Eugénie Granado, Emelyne Gervais, Guillaume Gotti, Sandrine Desclaux, Martine Meireles, \\ Pierre Gros, David Evrard* \\ Laboratoire de Génie Chimique, Université de Toulouse, CNRS, INPT, UPS, Toulouse, France \\ *E-mail: evrard@chimie.ups-tlse.fr
}

doi: $10.20964 / 2017.07 .44$

Received: 18 January 2017 / Accepted: 24 April 2017 / Published: 12 June 2017

\begin{abstract}
An electrochemical sensor dedicated to $\mathrm{Hg}(\mathrm{II})$ trace detection was elaborated based on a gold nanoparticles (AuNPs) modified glassy carbon (GC) electrode. AuNPs were prepared using the Turkevich method and deposited on GC by drop casting. Different protocols including suspension filtration and evaporation temperature control were tested and their influence both on coating morphology and electrochemical activity assessed. From structural characterization, it can be concluded that neither the filtration step nor the drop evaporating temperature have a significant impact on coating morphology at the mesoscale level. However, regarding to the electrochemical activity of the functionalized electrodes, results showed that when some heterogeneities due to (AuNPs) aggregation were present in the coating, the electrochemical activity was reduced. Contrary to what was observed in our previous studies dealing with electrodeposited AuNPs, cycling an AuNPsGC electrode in $\mathrm{H}_{2} \mathrm{SO}_{4}$ lead in all cases to a decrease in active surface area and in a higher density of surface defects, thus revealing a higher surface reactivity of chemically-synthesized AuNPs. The electrochemical activation procedure was found to influence the analytical performances of the functionalized electrode with respect to $\mathrm{Hg}(\mathrm{II})$ assay in the picomolar range, but not in the nanomolar range, as a consequence of a saturated concentration effect. A linear concentration range was obtained between 2 and $12 \mathrm{pM}$ with a normalized sensitivity of $0.296 \mu \mathrm{A} \mathrm{pM}^{-1} \mathrm{~min}^{-1}$ using square wave anodic stripping voltammetry (SWASV) as the detection mode. A limit of detection (LOD) down to $1 \mathrm{pM}$ was reached.
\end{abstract}

Keywords: Chemically prepared gold nanoparticles; drop-casting; deposit morphology; electrochemical reactivity; mercury(II) trace detection

\section{FULL TEXT}


(C) 2017 The Authors. Published by ESG (www.electrochemsci.org). This article is an open access article distributed under the terms and conditions of the Creative Commons Attribution license (http://creativecommons.org/licenses/by/4.0/). 